\title{
New frontiers in esophageal radiology
}

\author{
Eric J. Schmidlin ${ }^{1}$, Ritu R. Gill ${ }^{2}$ \\ ${ }^{1}$ Department of Radiology, Brigham and Women's Hospital, Harvard Medical School, Boston, MA, USA; ${ }^{2}$ Department of Radiology, Beth Israel \\ Deaconness Medical Center, Harvard Medical School, Boston, MA, USA \\ Contributions: (I) Conception and design: None; (II) Administrative support: Laura Patterson; (III) Provision of study materials or patients: None; \\ (IV) Collection and assembly of data: None; (V) Data analysis and interpretation: Both authors; (VI) Manuscript writing: Both authors; (VII) Final \\ approval of manuscript: Both authors. \\ Correspondence to: Eric J. Schmidlin, MD. Department of Radiology, Brigham and Women's Hospital, Harvard Medical School, 75 Francis St., \\ Boston, MA 02115, USA. Email: eschmidlin@bwh.harvard.edu.
}

\begin{abstract}
Esophageal cancer is the sixth most common cause of cancer related mortality worldwide. Advances in treatment have translated into steadily improving survival rates. Accurate preoperative staging of esophageal cancer is imperative in order to provide an accurate prognosis and direct patients to the most appropriate treatment. Current preoperative staging relies on imaging, most commonly endoscopic ultrasound (EUS), computed tomography (CT) and positron emission tomography (PET). A combination of these modalities should be used in preoperative staging, as each has advantages over another. Magnetic resonance imaging (MRI) has always shown promise in its ability to accurately stage esophageal cancer, though it has not been consistently adopted as a common tool for this purpose. Recent research has demonstrated that MRI can become an integral part of esophageal cancer clinical staging. Advances in MR technology that utilize radial sampling allow for shorter, free breathing techniques without degradation of image quality, resulting in improved capability for $\mathrm{T}$ and $\mathrm{N}$ staging of esophageal cancer. MRI enhanced with superparamagnetic iron oxide (SPIO) and ultrasmall SPIO (USPIO) nanoparticles has been shown to be useful for the detection of metastatic disease in lymph nodes. This article will review the current evidence in the role that imaging plays in staging esophageal cancer.
\end{abstract}

Keywords: Esophageal cancer; magnetic resonance imaging (MRI); computed tomography (CT); 2-fluoro-2deoxy-D-glucose positron emission tomography (FDG-PET)

Submitted Mar 27, 2020. Accepted for publication May 15, 2020.

doi: 10.21037/atm-20-2909

View this article at: http://dx.doi.org/10.21037/atm-20-2909

\section{Introduction}

Esophageal cancer is the sixth most common cause of cancer related mortality worldwide (1). It makes up approximately $1 \%$ of all cancers in the United States, but is much more common in China, India and Iran. While squamous cell carcinoma has long been the most common histologic type, adenocarcinoma has been consistently increasing in western countries. Advances in treatment have translated into steadily improving survival rates, with a $20 \% 5$-year survival for all stages of disease and $47 \%$ survival for localized disease in the United States (2). Accurate preoperative staging of esophageal cancer is imperative to determine prognosis and treatment. Staging relies on imaging, most commonly endoscopic ultrasound (EUS), computed tomography (CT) and positron emission tomography (PET). While magnetic resonance imaging (MRI) has always shown promise in its ability to accurately stage esophageal cancer, it has not been adopted as a common tool for this purpose. However, continued advancements in this imaging technology have demonstrated more promise than ever before in its ability to accurately stage esophageal cancer.

\section{Staging (TNM)}

The most recent $8^{\text {th }}$ edition of the American Joint 


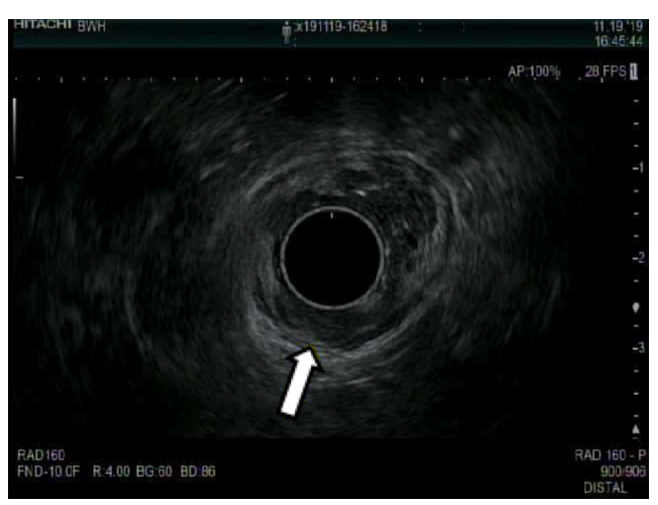

Figure 1 EUS image of the lower esophagus demonstrates a mass invading the muscularis submucosa (arrow), but not the muscularis propria in this patient with a T1b adenocarcinoma. EUS, endoscopic ultrasound.

Committee on Cancer (AJCC) staging of epithelial cancers of the esophagus and esophagogastric junction separates staging classifications into three groups, clinical (cTNM), pathologic (pTNM) and postneoadjuvant (ypTNM). In order to accurately reflect patient survival, separate groupings based on histologic cell type were created for both clinical and pathologic staging. While pathologic staging is the most accurate predictor of survival, clinical staging is paramount in determining which patients are likely to benefit from neoadjuvant therapy and esophagectomy. Postneoadjuvant staging (ypTNM) has been introduced by the AJCC, but its role in clinical practice is limited (3). Clinical staging is determined mostly by imaging and criteria include depth of tumor invasion, regional lymph node involvement and distant metastasis. CT has been the most frequently utilized modality for staging, though PET with 2-fluoro-2-deoxy-D-glucose (FDG) and EUS are now also commonly utilized. An approach utilizing a combination of all three modalities is now advocated for, as each modality has advantages over another in the staging workup.

\section{T classification}

The $T$ classification is determined by the depth of primary tumor invasion into the esophageal wall and by invasion of adjacent structures. T1 tumors invade the lamina propria or muscularis mucosa (T1a) or submucosa (T1b). T2 tumors invade the muscularis propria. T3 tumors invade the adventitia. T4 tumors invade structures adjacent to the esophagus and have been subcategorized into those that are still surgically resectable (T4a) and those that are generally not resectable (T4b). T4a tumors invade the pleura, pericardium, azygous vein, diaphragm or peritoneum. T4b tumors invade other structures such as the aorta, vertebral body or trachea/mainstem bronchus (3). Accurate clinical $\mathrm{T}$ staging (cT) is imperative, as it has important prognostic and treatment implications. cT1 and cT2 cancers are less likely to have nodal metastases and can be treated with surgical resection alone, where as cT3 and cT4 cancers are likely to have nodal metastases which would require neoadjuvant therapy (4).

\section{EUS}

EUS is the most accurate imaging modality in T staging of esophageal cancers, as it is able to distinguish the layers of the esophageal wall. The normal wall exhibits five alternating hyperechoic (white) and hypoechoic (black) layers. EUS can reliably distinguish between cT1/T2 disease, where there is not invasion beyond the muscularis propria, cT3 disease, where there is invasion beyond the muscularis propria and cT4 disease, where there is invasion beyond the adventitia (Figure 1). EUS has demonstrated a performance index of 0.89 at discriminating between $\mathrm{T} 1 / \mathrm{T} 2$ esophageal cancers from those that are T3 and T4 (5). EUS alone has not been shown to reliably distinguish between cT1a, cT1b and cT2 disease (6-9). EUS is also limited in its ability to accurately stage stenotic tumors that prohibit endoscope passage (10).

\section{$C T$}

Esophageal wall thickening is a non-specific sign of cancer on CT. A normal esophageal wall should always measure less than $5 \mathrm{~mm}$ (11), and less than $3 \mathrm{~mm}$ in a distended esophagus (12). Wall thickening is also commonly seen in esophagitis. The inability of CT to distinguish the layers of the esophageal wall limits its ability to accurately discriminate cT1, cT2 and cT3 tumors (Figure 2). However, CT is the most accurate imaging modality in assessing the presence of cT4 disease, which is excluded by demonstrating a preserved fat plane between tumor and adjacent structures (13). In addition to loss of adjacent fat planes, displacement or indentation of an adjacent structure is also an additional CT criterion for local invasion $(14,15)$. CT has demonstrated sensitivities and specificities ranging from $85-100 \%$ in detecting invasion of adjacent mediastinal 


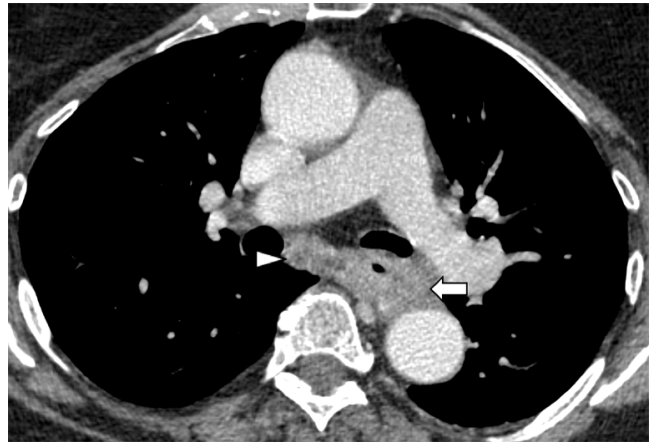

Figure 2 Axial contrast enhanced CT image demonstrates a midesophageal mass with circumferential wall thickening and an illdefined appearance of the adjacent fat in this patient with T3 disease (arrow). Note the enlarged subcarinal metastatic lymph node contributing to N2 disease (arrowhead). CT, computed tomography.

structures $(16,17)$.

\section{FDG-PET}

FDG-PET is more sensitive than CT in its ability to detect esophageal cancer (18). However, it has a very limited role in assessing $\mathrm{T}$ stage given its inability to accurately assess depth of tumor invasion.

\section{N classification}

Pathological nodal status is based on the presence (N1) or absence (N0) of regional periesophageal lymph node involvement. It is the most important prognostic factor in esophageal cancer staging (19). The number of involved nodes determines the $\mathrm{N}$ stage, with $\mathrm{N} 1$ disease involving 1-2 nodes, N2 disease involving 3-6 regional nodes and $\mathrm{N} 3$ involving 7 or more regional nodes. Clinical nodal classification $(\mathrm{cN})$ indirectly assesses the potential for a lymph node to harbor metastatic disease and primarily is accomplished by utilizing EUS, CT and FDG-PET, each of which has its own limitations.

\section{EUS}

EUS is able to evaluate the size, shape, border, cortical thickness and internal echotexture of reginal lymph nodes. Characteristics which make nodal involvement more likely include larger size, rounded morphology and a well-demarcated hypoechoic appearance. EUS is more accurate than CT in determining likelihood of lymph node involvement, with accuracy rates of $72-80 \%(20,21)$. However, EUS was only $20 \%$ specific in a more recent assessment that used criteria of $>5 \mathrm{~mm}$ in size, round border, smooth shape and hypoechoic center as indicators of lymph node involvement (22).

\section{$C T$}

CT depends primarily on size criteria, where intrathoracic and abdominal lymph nodes larger than $1 \mathrm{~cm}$ in short axis and supraclavicular lymph nodes larger than $0.5 \mathrm{~cm}$ are considered abnormal $(23,24)$. False negative assessments can occur in normal sized lymph nodes that contain metastatic disease and when positive lymph nodes are in close proximity to and obscured by the primary tumor (25). False positive assessments can occur when reactive lymph nodes become enlarged. CT is therefore less accurate at predicting lymph node involvement than EUS, with accuracy rates of $46-58 \%(20,21)$.

\section{FDG-PET}

FDG-PET is more specific than CT at predicting lymph node involvement, as it is able to detect both size and degree of hypermetabolic activity. In a meta-analysis FDGPET was $57 \%$ sensitive (range of $43-70 \%$ ) and $85 \%$ specific (range of 76-95\%) (26). The lower sensitivity may result from difficulty in distinguishing peritumoral lymph nodes that are obscured by the hypermetabolic primary tumor and from the presence of hypermetabolic reactive nodes. The high specificity of FDG-PET makes it an excellent modality to confirm cN0 disease.

Given the importance of accurate $\mathrm{cN}$ staging and the somewhat limited sensitivity or specificity of the imaging modalities, histologic confirmation of $\mathrm{cN}$ with EUS-guided fine-needle aspiration (FNA) is critical and is strongly recommended by the AJCC (27).

\section{M classification}

Hematogenous metastasis of esophageal cancer to distant organs defines the $M$ classification, designated as absent (M0) or present (M1). Early detection of distant metastatic disease is very important in determining the most appropriate treatment. The presence of metastatic disease has been reported in $20-30 \%$ of esophageal cancer patients, with liver, lungs and bones being the most commonly 

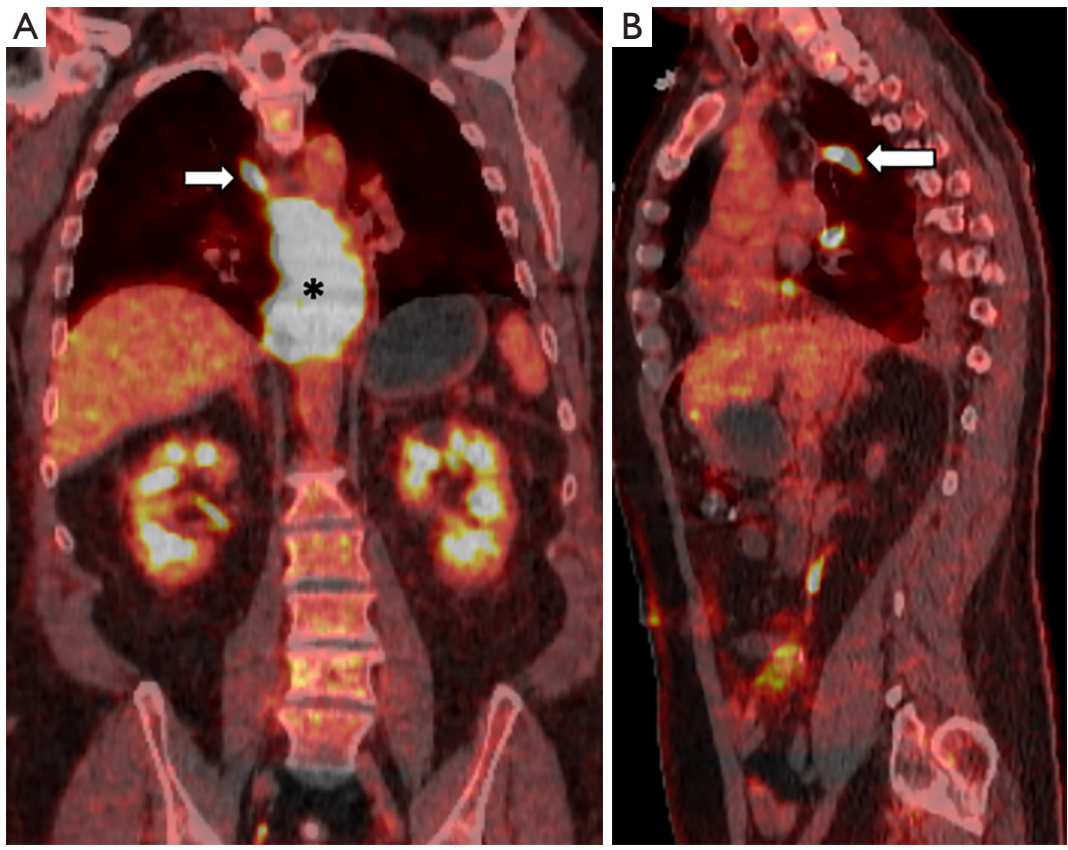

Figure 3 Coronal (A) and Sagittal (B) FDG-PET/CT fused image demonstrates a large mid and lower esophageal hypermetabolic mass extending beyond the adventitia (asterisk). There is also hypermetabolic tumor extending into and along the azygous vein in this patient with T4a adenocarcinoma (arrow). FDG-PET, 2-fluoro-2-deoxy-D-glucose positron emission tomography; CT, computed tomography.

involved organs $(28,29)$.

\section{EUS}

The value of EUS in screening for distant metastases is extremely limited, as the distant organ being evaluated must be in direct with the upper gastrointestinal tract.

\section{$C T$}

CT has long been the mainstay for assessment of metastatic esophageal cancer. It has the ability to detect metastasis in the most commonly affected organs, lungs, liver and bones. It has demonstrated a high specificity of $91 \%$, but a low sensitivity ranging from $37-66 \%(26,30)$.

\section{FDG-PET}

FDG-PET is able to detect additional sites of esophageal cancer metastasis not detected by conventional CT. It has the advantage over CT of offering both total body coverage and functional assessment, allowing detection of unsuspecting metastatic lesions (Figure 3). It has a similar specificity of $93 \%$, but markedly improved sensitivity of
71\% compared to CT (26). FDG-PET has been shown to affect $M$ stage in $24 \%$ of patients, upstaging from $M 0$ to $M 1$ disease in $22 \%$ and downstaging from $M 1$ to $M 0$ disease in $2 \%$ (31). PET does have limitations, including false positive findings in the setting of infection or inflammation and false negative results in types of esophageal cancer which fail to demonstrate hypermetabolic activity.

\section{MRI}

While a combination of EUS, CT and PET have become the standard imaging modalities utilized in esophageal staging, each has its limitations. Continued advancements in MRI have demonstrated more promise than ever before in its ability to accurately stage esophageal cancer. Advantages of MRI include total body coverage, lack of ionizing radiation and contrast agents with lower risk profiles and less restrictions of their use.

\section{Regional MRI}

Initial in vitro high-resolution MRI of resected esophageal specimens demonstrated the ability to differentiate 8 layers of the esophageal wall, which exhibit alternating low and 
high signal intensities from the mucosa to the adventitia (32). Early in vivo MRI using a high resolution T2-weighted sequence demonstrated three distinct layers of the esophageal wall, the intermediate signal mucosa, surrounded by the high signal intensity submucosa, followed by the low signal intensity muscularis propria. The high signal intensity periesophageal fat and structures within it were also clearly demonstrated. Esophageal cancers are most commonly of intermediate signal intensity, though tumors with fibrosis demonstrate lower signal intensity and mucinous tumors demonstrate high signal intensity. An initial proposal for MRI criteria for local staging was as follows: T1 exhibits no discernable tumor; $\mathrm{T} 2$ exhibits tumor within the submucosa and muscularis propria, but with an intact outer margin of the muscularis propria; T3 exhibits nodular irregularity of the outer margin of the muscularis propria and extension into the periesophageal fat; and T4 exhibits tumor extending into adjacent structures. It was demonstrated that MRI was able to accurately distinguish between T2 and T3 disease and could also clearly diagnose invasion of adjacent structures in T4 disease (33).

A prospective study comparing MRI with diffusionweighted imaging (DWI) to EUS, CT and PET in preoperative staging of esophageal cancer demonstrated that MRI showed the highest specificity (92\%) and positive predictive value $(80 \%)$ for T-staging, but with a reduced sensitivity of $67 \%$. EUS was the most sensitive (100\%) and had the highest negative predictive value $(100 \%)$ for T-staging. Both MR and EUS demonstrated a $100 \%$ sensitivity for $\mathrm{N}$-staging, though with very low specificities of $57 \%$ and $36 \%$ respectively (34).

MRI is susceptible to motion artifact, namely cardiac and respiratory motion in the case of esophageal cancer imaging. These limitations can be mitigated by applying cardiac and respiratory gating, though with resulting longer acquisition times. Advances in MR technology that utilize radial sampling allow for shorter, free breathing techniques without degradation of image quality (35). Two vendor specific sequences, T2-weighted turbo spinecho (TSE) BLADE and StarVIBE (Siemens Healthcare, Erlangen, Germany) were used for T-staging of esophageal cancer (Figure 4). A combination of these sequences after neoadjuvant chemotherapy was very accurate in correctly staging T1-T4 lesions, with areas under the curve (AUCs) of 0.886 for T1, 0.917 for T2, 0.943 for T3 and 0.930 for T4. The AUC for T0 tumors was the lowest at 0.667. The high-resolution delayed phase StarVIBE had the highest accuracy in correctly staging T0, T1, T2 and T4 tumors
(AUCs of $0.667,0.886,0.917$ and 0.930 respectively), while the T2-weighted TSE BLADE was the most accurate sequence for T3 tumors (AUC of 0.952) (36).

\section{Apparent diffusion coefficient (ADC)}

DWI is a MR sequence based on the motion of water molecules. The diffusion of water molecules, which is impaired in cancer cells, can be expressed quantitatively as ADC values. There is not a correlation between ADC values and pathologic types of esophageal cancer. However, a correlation between ADC value and histologic grade has been demonstrated, with poorly differentiated tumors exhibiting lower ADC values (37). The degree of change in $\mathrm{ADC}$ values before and after neoadjuvant therapy has been shown to correlate with response to treatment, which could potentially be used to determine response to treatment, similar to how standardized uptake values (SUVs) are currently used to measure response to treatment with PET $(38,39)$. There is conflicting evidence on whether pretreatment $\mathrm{ADC}$ values can predict response to neoadjuvant therapy, with one study demonstrating lower ADC values in patients that were likely to respond to neoadjuvant therapy, while others demonstrated higher ADC values predicted a good response (40-42).

\section{Nodal MRI}

MRI has been traditionally challenged in its ability to accurately predict nodal metastases given that it relies on size in making this determination. Most of the metastatic lymph nodes in esophageal cancer measure less than $1 \mathrm{~cm}$, below the typical imaging threshold used for suspected lymph node metastases (43). Utilizing a STIR TSE sequence with electrocardiogram (ECG) gating resulted in improved accuracy in predicting lymph node metastases, with a sensitivity of $81 \%$ and specificity of $98 \%$, compared to $36 \%$ sensitivity and $86 \%$ specificity with conventional MRI (44).

ADC values obtained from DWI MR can also improve detection of lymph node metastases, as ADC values are lower in metastatic than in benign lymph nodes $(45,46)$. DWI has been shown to offer similar specificity, but improved sensitivity (67\%) compared to conventional FDG-PET (32\%) in detecting metastatic lymph nodes in patients with squamous cell esophageal cancer (47).

MRI enhanced with superparamagnetic iron oxide (SPIO) and ultrasmall SPIO (USPIO) nanoparticles has been shown 

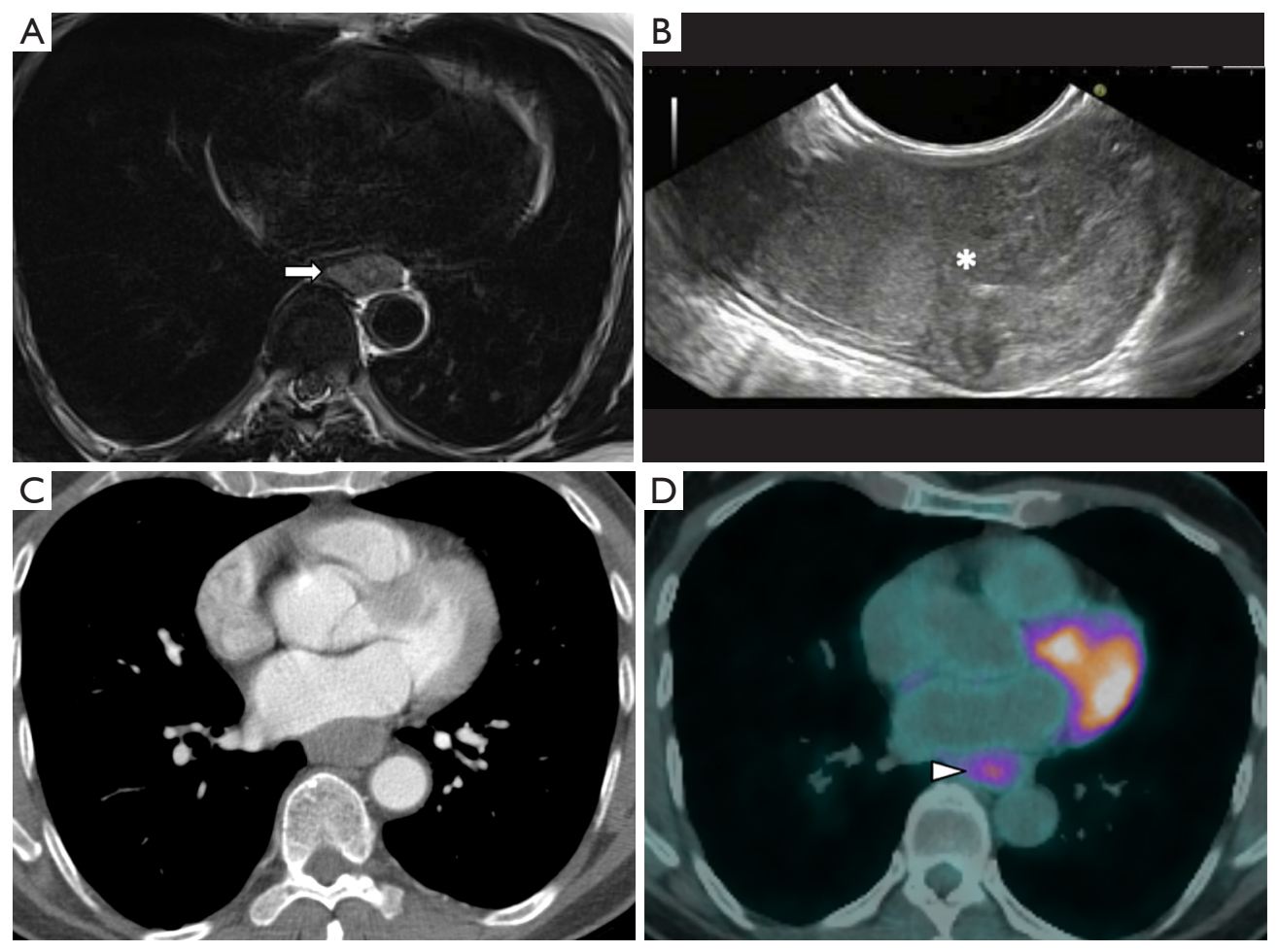

Figure 4 Axial MR T2 BLADE image (A) demonstrating a hyperintense mid-esophageal submucosal mass (arrow) which invades the muscularis propria, but not the adventitia, in this patient with T2 adenoid cystic carcinoma; (B) EUS image in the same patient demonstrates the submucosal mass is fairly homogeneous and well-circumscribed (asterisk); (C) axial contrast enhanced CT image demonstrates the mass does not invade in the peri-esophageal fat nor adjacent structures; (D) FDG-PET/CT fused image demonstrates the mass is metabolically avid (arrowhead) without metastatic disease. MR, magnetic resonance; FDG-PET, 2-fluoro-2-deoxy-D-glucose positron emission tomography; CT, computed tomography.

to be useful for the detection of metastatic tumors in lymph nodes, even in nodes that are not enlarged by traditional criteria. These nanoparticles are unable to phagocytosed by lymph nodes involved by tumor because they lack reticuloendothelial cells. As a result, they appear dark on $\mathrm{T} 2$-weighted images because of their superparamagnetic effect (48). Limited feasibility studies in esophageal cancer patients have demonstrated that this technique was able to accurately identify the majority of metastatic mediastinal and celiac axis lymph nodes (49).

\section{Total body MRI}

A total body MRI approach with tailored sequences to detect metastasis demonstrated the ability to detect $98 \%$ of primary esophageal lesions, compared to $96 \%$ for PET/ CT. This technique also demonstrated a sensitivity of $27 \%$, specificity of $100 \%$ and accuracy of $56 \%$ in detecting nodal metastases, compared to $30 \%, 100 \%$ and $60 \%$ respectively for PET/CT. Both modalities detected distant metastases in two patients $(42,50)$.

\section{PET/MR}

Previous studies have demonstrated conflicting results as to whether SUV measurements with FDG-PET can predict outcomes in patients with esophageal cancer (51-53). Integrated 18F-PET/MRI combines whole body MRI and PET, which can therefore provide functional information in the form of both ADC values from DWI and glycolytic activity from PET. This functional information has been shown to correlate with TNM staging in patients with esophageal carcinoma. The minimum ADC value exhibited the strongest inverse correlation with SUVs and was lower in higher T-stage tumors. Volume based parameters can also be calculated and include total lesion glycolysis and 
metabolic tumor volume, which were more accurate at predicting $\mathrm{T}$ - and $\mathrm{N}$-stage respectively, than were ADC values (54).

\section{MRI and response to neoadjuvant chemoradiotherapy}

Neoadjuvant chemoradiotherapy can result in a complete pathological response in $25-30 \%$ of patients with locally advanced esophageal cancer (55). These patients may not require surgical resection and identifying them is therefore important, though CT, FDG-PET/CT and EUS are limited in their ability to do so given the difficulty in discriminating residual tumor from radiation esophagitis and residual wall thickening (56). MRI utilizing both T2weighted and DWI sequences demonstrated a higher sensitivity in detecting residual disease at 90-97\%, though with a specificity of only $42-50 \%$ (57). Use of MRI alone would therefore result in many complete responders being misdiagnosed as having residual disease, and therefore a combination of modalities is therefore needed to correctly diagnose complete responders.

\section{Additional applications}

MRI has shown promise in its ability to assess for nonneoplastic diseases. Dynamic or functional MR can assess for gastroesophageal reflux disease, achalasia and other motility disorders (58-61). It can also be used to assess postoperative complications of esophageal surgery such as fundoplication (62). While it offers the advantages of lack of ionizing radiation and increased spatial resolution, the increased time, cost and less availability compared to traditional fluoroscopy make routine and widespread utilization of MRI for these purposes unlikely.

\section{Conclusions}

Preoperative staging and assessment of neoadjuvant treatment response in esophageal cancer most often relies on a combination of EUS, CT and/or PET. Each of these modalities has its advantages in being able to accurately determine TNM stage. MRI has always shown promise in its ability to accurately stage esophageal cancer, but it has not yet achieved widespread adoption for this purpose. Technological advancements in MRI have resulted in improved image quality and faster acquisition times and recent research has demonstrated that MRI can be an integral part in the clinical staging of esophageal cancer.

\section{Acknowledgments}

Funding: None.

\section{Footnote}

Provenance and Peer Review: This article was commissioned by the Guest Editor (Dr. Ory Wiesel) for the series "Innovations and Updates in Esophageal Surgery" published in Annals of Translational Medicine. The article was sent for external peer review organized by the Guest Editor and the editorial office.

Conflicts of Interest: Both authors have completed the ICMJE uniform disclosure form (available at http://dx.doi. org/10.21037/atm-20-2909). The series "Innovations and Updates in Esophageal Surgery" was commissioned by the editorial office without any funding or sponsorship. The authors have no other conflicts of interest to declare.

Ethical Statement: The authors are accountable for all aspects of the work in ensuring that questions related to the accuracy or integrity of any part of the work are appropriately investigated and resolved.

Open Access Statement: This is an Open Access article distributed in accordance with the Creative Commons Attribution-NonCommercial-NoDerivs 4.0 International License (CC BY-NC-ND 4.0), which permits the noncommercial replication and distribution of the article with the strict proviso that no changes or edits are made and the original work is properly cited (including links to both the formal publication through the relevant DOI and the license). See: https://creativecommons.org/licenses/by-nc-nd/4.0/.

\section{References}

1. Ferlay J, Soerjomataram I, Dikshit R, et al. Cancer incidence and mortality worldwide: sources, methods and major patterns in GLOBOCAN 20012. Int J Cancer 2015;136:E359-86.

2. American Cancer Society. Key statistics for esophageal cancer. Atlanta: the American Cancer Society, 2017. Available online: https://www.cancer.org/cancer/ esophagus-cancer/about/key-statistics.html (accessed 
2/20/20).

3. Rice TW, Patil DT, Blackstone EH. 8th edition AJCC/UICC staging of cancers of the esophagus and esophagogastric junction: application to clinical practice. Ann Cardiothorac Surg 2017;6:119-30.

4. Rice TW, Ishwaran H, Hofstetter WL, et al. Esophageal cancer: associations with $(\mathrm{pN}+)$ lymph node metastases. Ann Surg 2017;265:122-9.

5. Kelly S, Harris KM, Berry E, et al. A systematic review of the staging performance of endoscopic ultrasound in gastro-oesophageal carcinoma. Gut 2001;49:534-9.

6. Blackshaw G, Lewis WG, Hopper AN, et al. Prospective comparison of endosonography, computed tomography, and histopathological stage of junctional oesophagogastric cancer. Clin Radiol 2008;63:1092-8.

7. Murata Y, Nopleon B, Odegaard S. High-frequency endoscopic ultrasonography in the evaluation of superficial esophageal cancer. Endoscopy 2003;35:429-35.

8. Puli SR, Reddy JB, Bechtold ML, et al. Staging accuracy of esophageal cancer by endoscopic ultrasound: a metaanalysis and systematic review. World J Gastroenterol 2008;14:1479-90.

9. Rice TW, Mason DP, Murthy SC, et al. T2N0M0 esophageal cancer. J Thorac Cardiovasc Surg 2007;133:317-24.

10. Iyer RB, Silverman PM, Tamm EP, et al. Diagnosis, staging and follow-up of esophageal cancer. AJR. Am J Roentgenol 2003;181:785-93.

11. Desai RK, Tagliabue JR, Wegryn SA, et al. CT evaluation of wall thickening in the alimentary tract. Radiographics 1991;11:771-83.

12. Noh HM, Fishman EK, Forastiere AA, et al. CT of the esophagus: spectrum of disease with emphasis on esophageal carcinoma. Radiographics 1995;15:1113-34.

13. Rice TW. Clinical staging of esophageal carcinoma. CT, EUS and PET. Chest Surg Clin N Am 2000;10:471-85.

14. Takashima S, Takeuchi N, Shiozaki H, et al. Carcinoma of the esophagus: CT vs MR imaging in determining resectability. AJR Am J Roentgenol 1991;156:297-302.

15. Halvorsen RA Jr, Magruder-Habib K, Foser WL Jr, et al. Esophageal cancer staging by CT: long-term follow-up study. Radiology 1986;161:147-51.

16. Picus D, Balfe DM, Koehler RE, et al. Computed tomography in the staging of esophageal carcinoma. Radiology 1983;146:433-8.

17. Daffner RH, Halber MD, Postlethwait RW, et al. CT of the esophagus. II. Carcinoma. AJR Am J Roentgenol 1979;133:1051-5.
18. Block MI, Patterson GA, Sundaresan RS, et al.

Improvement in staging of esophageal cancer with the addition of positron emission tomography. Ann Thorac Surg 1997;64:770-6; discussion 776-7.

19. Lerut T, Coosemans W, Decker G, et al. Cancer of the esophagus and gastro-esophageal junction: potentially curative therapies. Surg Oncol 2001;10:113-22.

20. Kalantzis N, Kallimanis G, Laoudi F, et al. Endoscopic ultrasonography and computed tomography in preoperative (TNM) classification of oesophageal carcinoma. Endoscopy 1992;24:abstr 653A.

21. Souquet JC, Napoleon B, Pujol B, et al. Endoscopic ultrasonography in the preoperative staging of esophageal cancer. Endoscopy 1994;26:764-6.

22. Kutup A, Link BC, Schurr PG, et al. Quality control of endoscopic ultrasound in preoperative staging of esophageal cancer. Endoscopy 2007;39:715-9.

23. Dorfman RE, Alpern MB, Gross BH. Upper abdominal lymph nodes: criteria for normal size determined with CT. Radiology 1991;180:319-22.

24. Fultz PJ, Feins RH, Strang JG, et al. Detection and diagnosis of nonpalpable supraclavicular lymph nodes in lung cancer at CT and US. Radiology 2002;222:245-51.

25. Kumbasar B. Carcinoma of esophagus: radiologic diagnosis and staging. Eur J Radiol 2002;42:170-80.

26. van Vliet EP, Heijenbrok-Kal MH, Hunink MG, et al. Staging investigations for oesophageal cancer: a metaanalysis. Br J Cancer 2008;98:547-57.

27. Rice TW, Kelsen DP, Blackstone EH, et al. Esophagus and esophagogastric junction. In: Amin MB, Edge SB, Greene FL, et al. editors. AJCC Cancer Staging Manual, 8th ed. New York: Springer, 2017:185-202.

28. Quint LE, Hepburn LM, Francis IR, et al. Incidence and distribution of distant metastases from newly diagnosed esophageal carcinoma. Cancer 1995;76:1120-5.

29. Flanagan FL, Dehdashti F, Siegel BA, et al. Staging of esophageal cancer with $18 \mathrm{~F}$-flurordeoxy-glucose positron emission tomography. AJR Am J Roentgenol 1997;168:417-24.

30. Li Z, Rice Tw. Diagnosis and staging of cancer of the esophagus and esophagogastric junction. Surg Clin North Am 2012;92:1105-26.

31. You JJ, Wong KS, Darling G, et al. Clinical utility of 18F-flurodeoxyglucose positron emission tomography/ computed tomography in staging of patients with potentially resectable esophageal cancer. J Thorac Oncol 2013;8:1563-9.

32. Yamada I, Izumi Y, Kawano T, et al. Superficial esophageal 
carcinoma: An in vitro study of high resolution MR imaging at 1.5T. J Magn Reson Imaging 2001;13:225-31.

33. Riddell AM, Allum WH, Thompson JN, et al. The appearance of oesophageal carcinoma demonstrated on high-resolution, T2-weighted MRI, with histopathological correlation. Eur Radiol 2007;17:391-9.

34. Giganti F, Ambrosi A, Petrone MC, et al. Prospective comparison of MR with diffusion-weighted imaging, endoscopic ultrasound, MDCT and positron emission tomography-CT in the pre-operative staging of oesophageal cancer: results from a pilot study. Br J Radiol 2016;89:20160087.

35. Fellner C, Menzel C, Fellner FA, et al. BLADE in T2weighted MR imaging of the cervical spine. AJNR Am J Neuroradiol 2010;31:674-81.

36. Wang Z, Guo J, Qin J, et al. Accuracy of 3-T MRI for preoperative $\mathrm{T}$ staging of esophageal cancer after neoadjuvant chemotherapy, with histopathologic correlation. AJR Am J Roentgenol 2019;212:788-95.

37. Wang Y, Bai G, Guo L, et al. Associations between apparent diffusion coefficient value with pathological type, histologic grade, and presence of lymph node metastases of esophageal carcinoma. Technol Cancer Res Treat 2019; 18:1533033819892254.

38. van Rossum PS, van Lier AL, van Vulpen $M$, et al. Diffusion-weighted magnetic resonance imaging for the prediction of pathologic response to neoadjuvant chemoradiotherapy in esophageal cancer. Radiother Oncol 2015;115:163-70.

39. Chen YJ, Pu YS, Chueh SC, et al. Diffusion MRI predicts transrectal ultrasound biopsy results in prostate cancer detection. J Magn Reson Imaging 2011;33:356-63.

40. De Cobelli F, Giganti F, Orsenigo E, et al. Apparent diffusion coefficient modifications in assessing gastroesophageal cancer response to neoadjuvant treatment: comparison with tumor regression grade at histology. Eur Radiol 2013;23:2165-74.

41. Kwee RM, Dik AK, Sosef MN, et al. Interobserver reproducibility of diffusion-weighted MRI in monitoring tumor response to neoadjuvant therapy in esophageal cancer. PLoS One 2014;9:e92211.

42. Aoyagi T, Shuto K, Okazumi S, et al. Apparent diffusion coefficient values measured by diffusion-weighted imaging predict chemoradiotherapeutic effect for advanced esophageal cancer. Dig Surg 2011;28:252-7.

43. Kajiyama Y, Tsurumaru M, Iwanuma Y, et al. Controversies in Esophageal Cancer Surgery. Gan To Kagaku Ryoho 2003;30:1225-9.
44. Alper F, Turkyilmaz A, Kurtcan S, et al. Effectiveness of the STIR turbo spin-echo sequence MR imaging in evaluation of lymphadenopathy in esophageal cancer. Eur J Radiol 2011;80:625-8.

45. Lin G, Ho KC, Wang JJ, et al. Detection of lymph node metastasis in cervical and uterine cancers by diffusionweighted magnetic resonance imaging at 3T. J Magn Reson Imaging 2008;28:128-35.

46. Holzapfel K, Duetsch S, Fauser C, et al. Value of diffusionweighted MR imaging in the differentiation between benign and malignant cervical lymph nodes. Eur J Radiol 2009;72:381-7.

47. Shuto K, Kono T, Shiratori T, et al. Diagnostic performance of diffusion-weighted magnetic resonance imaging in assessing lymph node metastasis of esophageal cancer compared with PET. Esophagus 2020;17:239-49.

48. Corot C, Robert P, Idee JM, et al. Recent advances in iron oxide nanocrystal technology for medical imaging. Adv Drug Deliv Rev 2006;58:1471-504.

49. Pultrum BB, van der Jagt EJ, van Westreenen HL, et al. Detection of lymph node metastases with ultrasmall superparamagnetic iron oxide (USPIO)-enhanced magnetic resonance imaging in oesophageal cancer: a feasibility study. Cancer Imaging 2009;9:19-28.

50. Will O, Purkayastha S, Chan C, et al. Diagnostic precision of nanoparticle enhanced MRI for lymph node metastases: a meta-analysis. Lancet Oncol 2006;7:52-60.

51. Pan L, Gu P, Huang G, et al. Prognostic significance of SUV on PET/CT in patients with esophageal cancer: a systematic review and meta-analysis. Eur J Gastroenterol Hepatol 2009;21:1008-15.

52. Chatterton BE, Shon IH, Baldey A. Positron emission tomography changes management and prognostic stratification in patients with oesophageal cancer: results of a multicenter prospective study. Eur J Nucl Med Mol Imaging 2009;36:354-61.

53. Omloo JM, Sloof GW, Boellaard OS, et al. Importance of FDG-PET and endoscopic ultrasound parameters in predicting survival following surgery for esophageal cancer. Endoscopy 2008;40:464-71.

54. Yu CW, Chen XJ, Lin YH, et al. Prognostic value of 18F-FDG PET/MR imaging biomarkers in oesophageal squamous cell carcinoma. Eur J Radiol 2019;120:108671.

55. van Hagen P, Hulshof MC, van Lanschot JJ, et al. Preoperative chemoradiotherapy for esophageal or junctional cancer. N Engl J Med 2012;366:2074-84.

56. Yip C, Cook GJ, Landau DB, et al. Performance of different imaging modalities in assessment of response to 
neoadjuvant therapy in primary esophageal cancer. Dis Esophagus 2016;29:116-30.

57. Vollenbrock SE, Voncken FEM, van Dieren JM, et al. Diagnostic performance of MRI for assessment of response to neoadjuvant chemoradiotherapy in oesophageal cancer. Br J Surg 2019;106:596-605.

58. Kulinna-Cosentini C, Schima W, Cosentini EP. Dynamic MR imaging of the gastroesophageal junction in healthy volunteers during bolus passage. J Magn Reson Imaging 2007;25:749-54.

59. Curcic J, Fox M, Kaurfman E, et al. Gastroesophageal junction: structure and function as assessed by using MR imaging. Radiology 2010;257:115-24.

60. Panebianco V, Tomei E, Anzidei M, et al. Functional

Cite this article as: Schmidlin EJ, Gill RR. New frontiers in esophageal radiology. Ann Transl Med 2021;9(10):904. doi: 10.21037/atm-20-2909
MRI in the evaluation of oesophageal motility: feasibility, MRI patterns of normality, and preliminary experience in subjects with motility disorders. Radiol Med 2006;111:881-9.

61. Panebianco V, Habib FI, Tomei E, et al. Initial experience with magnetic resonance fluoroscopy in the evaluation of oesophageal motility disorders. Comparison with manometry and barium fluoroscopy. Eur Radiol 2006;16:1926-33.

62. Arnoldner MA, Kristo I, Paireder M, et al. Swallowing MRI-a reliable method for the evaluation of the postoperative gastroesophageal situs after Nissed fundoplication. Eur Radiol 2019;29:4400-7. 